\title{
A Predictive Model of Anesthesia Depth Based on SVM in the Primary Visual Cortex
}

\author{
Li Shi, Xiaoyuan $\mathrm{Li}^{*}$ and Hong Wan
}

School of Electrical Engineering, Zhengzhou University, Zhengzhou, Henan, China

\begin{abstract}
In this paper, a novel model for predicting anesthesia depth is put forward based on local field potentials (LFPs) in the primary visual cortex (V1 area) of rats. The model is constructed using a Support Vector Machine (SVM) to realize anesthesia depth online prediction and classification. The raw LFP signal was first decomposed into some special scaling components. Among these components, those containing higher frequency information were well suited for more precise analysis of the performance of the anesthetic depth by wavelet transform. Secondly, the characteristics of anesthetized states were extracted by complexity analysis. In addition, two frequency domain parameters were selected. The above extracted features were used as the input vector of the predicting model. Finally, we collected the anesthesia samples from the LFP recordings under the visual stimulus experiments of Long Evans rats. Our results indicate that the predictive model is accurate and computationally fast, and that it is also well suited for online predicting.
\end{abstract}

Keywords: Anesthesia Depth, Local Field Potential, Complexity Analysis, Wavelet Transform, Support Vector Machine.

\section{INTRODUCTION}

Microelectrode array implant technology is usually adopted in the neurophysiological studies of animals. Physiological studies show that the anesthesia depth influences the physiological function of animals and signal acquisition considerably [1]. Accurate prediction of the anesthesia depth is very important to maintain the stable anesthesia state and ensure consistency of the experiment conditions. Therefore, building a predictive anesthesia model is of great value for scientific research and clinical application as it can offer a new method for monitoring anesthetic levels during surgery.

It has been well known that anesthetics alters the brain's neuronal activities and causes changes in electrical signals $[2,26]$. Thus, the characterization of the electroencephalogram (EEG) analysis becomes one of the main ways for monitoring the anesthesia depth $[3,4]$. Electrical activities of the brain mainly include EEG and local field potentials (LFPs). The former is the spontaneous activity recorded from the surface of the scalp, and the latter is recorded on the brain surface or within the brain. The earliest application using EEG signals to monitor the anesthesia depth began in 1940 [5]. A large number of efforts have been made to develop and test various EEG-derived parameters in different analysis methods $[4,6]$. However, these time, frequency and bispectral- domain methods have not considered the nonlinear dynamic behavior of the EEG and complexity of the anesthetic effect. The monitoring efficiency is affected by sensitivity and specificity decline due to different individuals

*Address correspondence to this author at the School of Electrical Engineering, Zhengzhou University, Zhengzhou 450001, No.100, Ke-Xue Rd., PR China; Tel: 86-13613717322; Fax: +86-0371-67783113;

E-mail: lixiaoyuan@zzu.edu.cn
[7-9]. The bispectral index scale (BIS) [10, 11] effectively predicts the status of anesthetic metabolism, but neither the transition change from being awake to unconsciousness nor the prediction of the anesthesia depth for anesthetics compound is well assessed. Another method in the clinical application is the Narcotrend index (NI), which guides us to reduce the consumption of anesthetic and shortens recovery time in the process of anesthesia [12]. Both of these depend obviously on anesthetic and neither can reflect analgesic composition in different anesthesia depths. The approaches of monitoring the anesthesia depth include the multi-layer neural network [13] and the fuzzy neural network [14], although these methods have not considered the non-linear and dynamical properties of the brain's neuronal activities. There are many problems to be solved in relation to clinical operations [15]. Recent advances suggest that electrical activity is a manifest of the brain's complicated nonlinear dynamical properties [16]. Therefore, nonlinear dynamical analysis methods, such as approximate entropy [17], Kolmogorov entropy [18], and combination entropy [19], have been widely used for feature extraction of the anesthesia depth. However, these methods are computationally expensive and are not satisfactory in the case of online monitoring of clinical operations.

There are three causes limiting the accuracy of predicting the state of anesthesia with the EEG. Firstly, EEG signals only represent the integration of function activities in the different cortical areas and do not reflect the functional conditions under cortical organization. The EEG based monitoring methods mentioned above are not sensitive to noxious stimuli [4]. Secondly, the EEG signals are sensitive to noisy overlapping, especially potential electromyographic interferences. Finally, the frequency band of the EEG, always below $30 \mathrm{~Hz}$, restricts the application at higher frequencies, such as 
the $\gamma$-band, which supplies more information for precise analysis of the anesthesia depth [20]. LFPs recorded from intracranial techniques represent a summation of the multiple action potentials in the extracellular space around neurons and reflect to noxious stimuli more effectively. Matthias Kreuzer indicated that LFPs form different channels which might cause excessive synchrony with the deepening of anesthesia and could provide additional information for detecting the levels of anesthesia [21]. Hudetz found that the crossapproximate entropy (XApEn) derived from LFPs is similar to the result deduced from EEG in different cortical areas $[22,23]$. In addition, LFPs are less contaminated by the electromyographic activity and can offer higher frequency information which can reflect the effect of anesthesia accurately [24].

According to the purpose of study the neural mechanism of visual pathway, the microelectrode array implant technology is adopted to record LFP signals in primary visual cortex(V1) of rats. Accurate prediction of the anesthesia depth is very important to maintain the stable anesthesia state in order to ensure consistency of experiment condition and determine the suitable time to supply the anesthetic. Therefore, a predictive model of anesthesia on the basis of LFPs is put forward in this paper. It is constructed using a Support Vector Machine (SVM) to realize anesthesia prediction in real time. First of all, taking into account the raw LFPs' non-analytical, non-linear and non-stationary properties, the LFPs were decomposed into special scaling components which contained higher frequency information well suited for more precise analysis of the anesthetic depth by wavelet transform. The influence on different scaling components was analyzed for different anesthesia depths. Secondly, LempeI-Ziv complexity measures were extracted by complexity analysis in order to achieve online predictive anesthesia depth. At the same time, two frequency-domain parameters were introduced as the criteria to improve prediction precision. All of the characteristics extracted above formed the input vector of the model that used cross validation $(\mathrm{K}-\mathrm{CV})$ to optimize model parameters. Finally, the predicting model was acquired through samples training from LFPs recorded in the visual stimulus experiments of Long Evans rats. The results demonstrated that this model can predict the anesthesia depth accurately in real time and illustrated that anesthesia might impede proper information exchange between cortical areas [25], impair information integration function of the brain and suppress consciousness $[28,29]$. Our results are in agreement with previous studies.

\section{MATERIALS AND METHODS}

\subsection{Surgery and LFP Data}

Six adult Long Evans rats weighing $260 \pm 20 \mathrm{~g}$ were used in this study. The rats were maintained on a $12 \mathrm{~h}$ light, $12 \mathrm{~h}$ dark cycle, with free access to food and water. Food was deprived one day before the experiments. This study was approved specifically by the Animal Care and Use Committee of the Zhengzhou University, and conducted in the Animal Center of Henan Province. After receiving atropine sulfate $(0.02-0.05 \mathrm{mg} / \mathrm{kg}$, intravenously (IV)), the rats were anesthetized with ketamine hydrochloride $(50 \mathrm{mg} / \mathrm{kg}$ initially, maintained at $10 \mathrm{mg} / \mathrm{hr}, \mathrm{IV}$ ) and were secured in a stereotaxic apparatus. Additional ketamine hydrochloride was added upon the anesthetic level and the stability of LFPs were recorded in the vision stimulation experiments. Body temperature was maintained at $37^{\circ} \mathrm{C}-38^{\circ} \mathrm{C}$ with a thermostatically controlled heating pad. The heart rate was monitored continuously. A craniotomy was made over the V1 area (coordinates: $0-4 \mathrm{~mm}$ anterior to lambda and $2.5-4.5 \mathrm{~mm}$ lateral to the midline) and the dura was removed under a highmagnification dissecting microscope. LFPs were obtained through microelectrode array ((MEA), Microprobe $2 \times 8$, impedance $0.50 \sim 1.0 \mathrm{M} \Omega$ ) implanted in Long Evans rats' V1 and recorded through a Cerebus 128-channel acquisition system (Blackrock microsystems, Salt Lake, USA). The microelectrode array was aimed at layer 4 in the V1 area. The LFP signals recorded from the electrodes were preamplified, filtered $(0-250 \mathrm{~Hz})$ and digitized at $2 \mathrm{kHz}$.

\subsection{Auxiliary Decision Criteria of Anesthesia State}

Although in recent years the anesthesia depth monitor based on brain electrical activities has developed rapidly, clinical signs and symptoms are still the basic method of auxiliary anesthesia depth judgment [31]. Among them, heart rate and tail flick that assess pain level (response to noxious stimuli), were used to estimate anesthesia depth [32]. These two parameters were sampled every 20 s and 60 s, respectively, and were recorded for six rats after small doses of supplement anesthetic injection. In this study, we considered tail flick latency $T s<5 \mathrm{~s}$ and heart rate $H R>380$ beat/min as the standard of a 'light' anesthesia state, and the corresponding tail flick latency $T s>8 \mathrm{~s}$ and heart rate $H R<$ $360 \mathrm{beat} / \mathrm{min}$ as the criterion of a 'deep' anesthesia state. The remaining period was considered as a transition state (including induction phase and recovery phase). In the whole experiment process, the 'light' anesthesia phase was the period of about 0s-600s after supplement anesthetic, and the 'deep' anesthesia phase was the period of about $600 \mathrm{~s}-2170 \mathrm{~s}$. Then, the recovery phase (2170s-) was also considered as a 'light' anesthesia state (as shown in Fig. 2).

\subsection{Analysis Methods}

\subsubsection{Channel Selection}

We utilized the cross-correlation function to measure their synchronization between different channels in order to quantify the effects of signal independence in different levels of anesthesia.

Pearson cross correlation coefficient is defined as

$$
r=\frac{\sum_{i=1}^{n}\left(x_{i}-\bar{x}\right)\left(y_{i}-\bar{y}\right)}{\sqrt{\sum_{i=1}^{n}\left(x_{i}-\bar{x}\right)^{2} \sum_{i=1}^{n}\left(y_{i}-\bar{y}\right)^{2}}}
$$

where $\bar{x}$ and $\bar{y}$ are the mean of the intervals $x=\left[x_{1}, x_{2}, \cdots x_{n}\right]$ and $y=\left[y_{1}, y_{2}, \cdots y_{n}\right]$.

\subsubsection{Building a Predictive Anesthetized State Model Based on Local Field Potentials in Primary Visual Cortex}

LFPs contain enough information to reliably predict the anesthetized state; however, the key problem is to construct a 
reasonable predictive model, extract the effective features and establish statistical training samples based on LFPs to realize classification accurately. We set up an SVM model to forecast the anesthetized states because of its many advantages in solving high dimensional and nonlinear pattern recognition problems through finite samples of SVM.

The designed prediction system consisted of an SVM classifier for off-line training and an on-line prediction system. LFPs had to be recorded from various states of the Long Evans rats and a specific database had to be built for off-line training before the system went into operation. These training samples can be updated in time in accordance with actual condition. Input vector $X_{k}=[X(1) \vee X(2), \cdots X(n)]$ was the feature vector extracted to represent the anesthesia depth from the $k$ th LFP segment. The feature extraction algorithm will be explained in detail in section Characters' Extraction. Output $Y(k+1)$ was the prediction result (slight anesthesia, Label 0 and deep anesthesia, Label 1) of the model in the $(k+1)$ th sample period.

To separate the set of training samples into two separate classes, let

$D=\left\{\left(X_{i}, Y_{i}\right) \mid X_{i} \in R^{n}, Y_{i} \in\{-1,1\}\right\}_{i=1}^{l}$

$Y_{i} \in\{-1,1\}$

In order to keep the balance between experience risk and generalization, non-negative variables $\xi_{i}$ were introduced to form a penalty function, $c \sum_{i=1}^{l} \xi_{i}$, where $\xi_{i}$ is a measure of the misclassification errors. The optimization problem was converted into maximizing the functional by the saddle point of the Lagrangian maximize

$Q(\alpha)=\sum_{i=1}^{l} \alpha_{i}-\frac{1}{2} \sum_{i, j=1}^{l} \alpha_{i} \alpha_{j} Y_{i} Y_{j}\left(X_{i} \cdot X_{j}\right)$

with constraints

$\sum_{i=1}^{l} Y_{i} \alpha_{i}=0$

$0 \leq \alpha_{i} \leq c \quad(i=1,2, \cdots, l)$

where $\alpha_{i}$ is the Lagrange multipliers.

An inner product in feature space has an equivalent kernel in input space,

$K\left(X_{i}, X_{j}\right)=\left\langle\Phi\left(X_{i}\right), \Phi\left(X_{j}\right)\right\rangle$

provided certain conditions hold. If $K$ is a symmetric positive definite function, which satisfies Mercer's Conditions, then the kernel represents a legitimate inner product in feature space. Suppose the inner product $\Phi\left(X_{i}\right) \cdot \Phi\left(X_{j}\right)$ is applied in the training algorithm, the subject function is

Maximized $Q(\alpha)=\sum_{i=1}^{l} \alpha_{i}-\frac{1}{2} \sum_{i, j=1}^{l} \alpha_{i} \alpha_{j} Y_{i} Y_{j} K\left(X_{i} \cdot X_{j}\right)$
The corresponding classification function also becomes

$$
\begin{aligned}
& f(X)=\operatorname{sgn}\left\{w \Phi(X)+b-1+\xi_{i}\right\} \\
& =\operatorname{sgn}\left\{\sum_{i=1}^{l} \alpha_{i}\left\{Y_{i} K\left(X_{i}, X_{j}\right)+b-1+\xi_{i}\right\}\right\}
\end{aligned}
$$

Kernel $K\left(X_{i}, X_{j}\right)$ is an inner product between input vector $X_{i}$ and support vector $X_{j}$; it can be chosen from many different mappings according to the requirements. The crossvalidation method was used here for optimizing the two variables, penalty factor $c$ and parameter $g$ of the kernel.

\subsubsection{Characters' Extraction}

\subsubsection{Decomposition of Raw LFPs by Wavelet Transform}

A raw LFP signal was decomposed into six consecutive scaling components $(\delta 0.3-3.9 \mathrm{~Hz}, \theta 3.9-7.8 \mathrm{~Hz}, \alpha$ 7.8-15.625 $\mathrm{Hz}, \beta 15.625-31.25 \mathrm{~Hz}, \gamma_{1} 31.25-46.8 \mathrm{~Hz}$ and $\gamma_{2} 46.8 \quad-$ $125 \mathrm{~Hz}$ ) by nine decomposition orders in order to analyze the effects of different frequency spectra under anesthesia. The orthogonal wavelet decomposition formula of signal $f(t)$ is given by the following equation

$$
\left\{\begin{array}{l}
c_{j, k}=\sum_{n} c_{j-1, n} h_{n-2 k} \\
d_{j, k}=\sum_{n} d_{j-1, n} g_{n-2 k}(k=0,1,2 \cdots N-1)
\end{array}\right.
$$

where $c_{j, k}$ and $d_{j, k}$ are the scaling coefficients and wavelet coefficients, respectively. $h$ and $g$ are called the filter group, $j$ is the number of layers of decomposition and $N$ is the number of data points in the signals.

The fast algorithm of Mallat's discrete dyadic wavelet transform is defined as

$c_{j-1, n}=\sum_{n} c_{j, n} h_{k-2 n}+\sum_{n} d_{j, n} g_{k-2 n}$

\subsubsection{Lempel-Ziv Complexity (LZC) Analysis of LFP}

In Lempel-Ziv's paper, complexity analysis, which uses nonlinear dynamics analysis, was adapted to characterize the information of electrical activities of the brain and to reveal the intrinsic nature under anesthesia. It is based on a coarse graining of the measurements and is well suited for clinical implementation due to its high computational speed [27].

Before calculating the complexity measure LZC of the LFP signal or its scaling components, described as $f$, the digital signal is first transformed into a sequence $f_{\min }$ whose elements are only a few symbols. Here, we only consider it as a $0-1$ string according to the mean value of the data points $f_{\max } \cdot f_{S E F}$ measures the number of distinct patterns that must be copied to reproduce a given sequence. Briefly, a sequence $P$ is scanned from left to right, and $f_{G F}$ is increased by one unit every time a new subsequence of consecutive digits is encountered in the scanning process.

In order to obtain a complexity measure which is independent of the sequence length, we used a normalized complexity measure. In reference [20], it has been proved that for 
a $0-1$ sequence, while $n \rightarrow \infty$, the upper bound of $C(n)$ is given by

$\lim _{n \rightarrow \infty} C(n)=b(n)=\frac{n}{\log _{2} n}$

and $C(n)$ can be normalized via

$L Z C=\frac{C(n)}{b(n)}$

The normalized complexity measure reflects the rate of new pattern occurrences with time and is usually less than one. It is independent of the amplitude and the length of the signal.

In this study, the LFPs were re-sampled at $500 \mathrm{~Hz}$ in order to improve the calculation speed and an LZC was calculated for every 10 s' LFP signals.

\subsubsection{Frequency Domain Analysis of LFP}

The $\delta$ ratio value and $\beta$ ratio value were extracted in the frequency domain as auxiliary parameters of the characteristic vector for predicting anesthesia depth. They are defined in equations (12) and (13), respectively.

$$
\begin{gathered}
\delta \quad \text { ratio }=\frac{P_{\delta}(0.3-3.9 \mathrm{~Hz})}{P_{\alpha}(7.8-15.6 \mathrm{~Hz})+P_{\beta}(15.6-31.25 \mathrm{~Hz})} \\
\beta \quad \text { ratio }=\log \left(P_{x x}(31-48 \mathrm{~Hz}) / P_{x x}(7.8-16 \mathrm{~Hz})\right)
\end{gathered}
$$

The variations of these two frequency domain parameters indicate that the deepening anesthesia depth can impair the peripheral nerve impulse conduction, enhance synchronization wave signals' promotion, and dampen nonsynchronization waves after the anesthetic injection. Therefore, these two parameters were introduced into the input vector to improve the precision of estimation.

\subsubsection{Establishing Training Samples Database}

According to the above requirements for constructing the forecasting model and characteristic vector extraction, LFPs were chosen from six experimental rats in the V1 area under different anesthesia states. After the signal's pretreatment, a total of 138 training data sets (including 59 samples in slight anesthesia and 79 samples in deep anesthesia), and 139 testing data sets (slight anesthesia 60, deep anesthesia 79) were obtained. Each training sample included the characteristic vector derived from LFP and the corresponding anesthesia state.

\subsection{Statistical Analysis}

Kruskal-Wallis' non-parametric test was introduced to analyze statistically significant differences in complexity measure LZC between the light anesthesia and deep anesthesia states. This was followed by multiple comparisons using the Mann-Whitney rank sum test to identify where the differences lay. A probability value of less than 0.05 was considered statistically significant. Data analysis was implemented in the Matlab development environment using both built-in functions and functions developed in-house.

\section{RESULTS}

\subsection{The Correlation of the LFPs Between the Recording Channels Under Different Anesthesia Depths}

LFPs recorded for $10 \mathrm{~s}$ in different periods after anesthetic injection are shown in Fig. (1). The top and bottom plots represent slight anesthesia and deep anesthesia, respectively. With the increasing anesthesia depth, the amplitude of low-frequency signal components was enhanced greatly and the amplitude of high frequency components was reduced, as shown in Fig. (1) (A, B, C and D). We found that the coherences between any two channels in deep anesthesia state were higher than those in slight anesthesia as a whole (see Figs. 1E and 1F). In particular, cross-correlation values of complexity measure of the LFPs were significantly higher than those of the LFPs. For example, the cross correlation value between the LFPs of channel 7 and channel 8 was 0.616 , but the cross-correlation value of complexity measure was 0.9454 . This illustrates that the complexity measure extracted from LFP has greater relevance to the anesthesia state, and that it can describe brain activities satisfactorily $[20,21,25]$.

\subsection{Complexity Measure of LFPs Under Different Anes- thesia Depth}

The Complexity measure LZC adopted in the paper is very simple and fast. The average calculation time of $L Z C$ for 10s LFP can satisfy the requirements of online operation.

The brain's activities are suppressed severely and its LFPs are dominated by low frequency waves in deep anesthesia. Consequently, LZC declined accordingly. It can be observed that $L Z C$ worked well in tracking the level of anesthesia, especially at the state-turning points (from light anesthesia depth to deep state or vice versa). Fig. (2) describes LZC against time for a rat's LFP recorded separately in the 16 channels during the anesthesia process.

\subsection{Comparison of Anesthetic Depth Indexes}

There are several indexes of anesthetic depth developed to translate the information of the complex LFP signals into a number. The spectral edge frequency(SEF) that consists of the power spectrum in the $95 \%$ quantile is a popular measure used to estimate the depth of anesthesia. Gravity Frequency $(\mathrm{GF})$ is another measure which can reflect the migrational features of signal energy during the process of anesthesia. Two calculation formula are defined as follows (14) and (15) respectively. $f_{G F}=\frac{\sum_{f=f_{\min }}^{f=f_{\max }}[P(f) \times f]}{\sum_{f=f_{\max }} P(f)}$

$$
\begin{aligned}
& \sum_{f=f_{\min }}^{f=f_{\text {SEF }}} P(f) \cdot * f=0.95 * E_{\text {total }} \\
& E_{\text {total }}==\sum_{f=f_{\min }}^{f=f_{\max }} P(f) \cdot * f
\end{aligned}
$$


A 20s-30s of LFPs after ketamine intravenously (light anesthesia state)

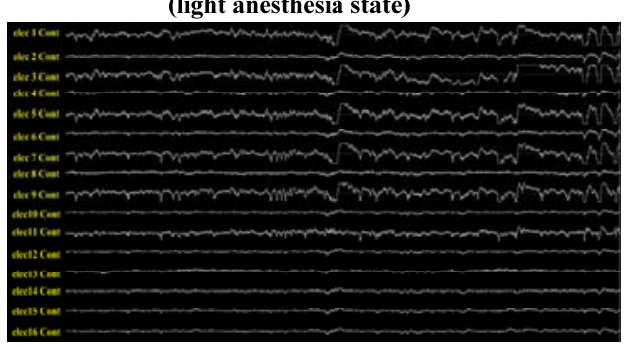

B 1000s-1010s of LFPs after ketamine intravenously (deep anesthesia state)

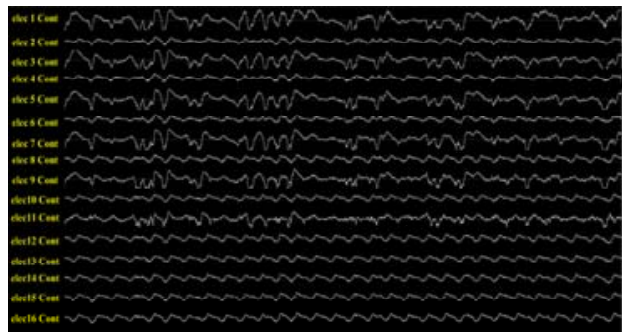

$1 \mathrm{mV}$

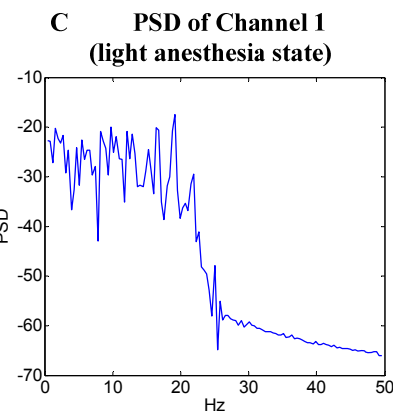

D PSD of Channel 1 (deep anesthesia state)

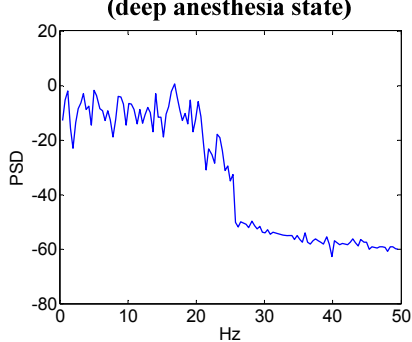

E Cross correlation of LFPs (light anesthesia state)

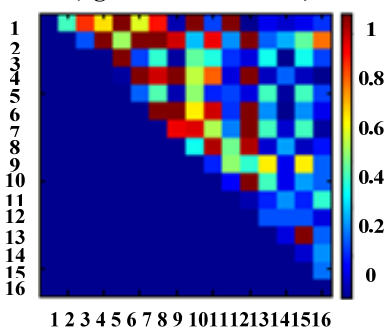

F Cross correlation of LFPs (deep anesthesia state)

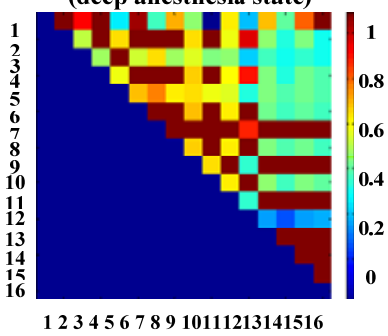

Fig. (1). The signal characteristics of neocortical local field potentials in V1 area. (A) and (B) 10s example of raw local field potentials from 16 electrodes, filtered between 0.3 and $250 \mathrm{~Hz}$, from the same rat under two different anesthesia states. 20s-30s LFP segments were illustrated in (A) after ketamine intravenously, and the rat was considered in light anesthesia state during this period of time. 1000s-1010s LFP segments were illustrated in (B) after ketamine intravenously, and the rat was considered in deep anesthesia state during this period of time. (C) Power spectral density (PSD) in light anesthesia state plots of all raw data segments(shown in A) selected for analysis (only channel1). (D) Power spectral density (PSD) in deep anesthesia state plots of all raw data segments (shown in B) selected for analysis in the same channel. (E) Cross correlation of the raw LFP segments of 16 chnnels depicted in A. Each color-coded matrix contains the values obtained from non-redundant pairwise combinations of recording sites (except auto-combinations). (F) Cross correlation of 16 chnnels in deep anesthesia state (depicted in B).

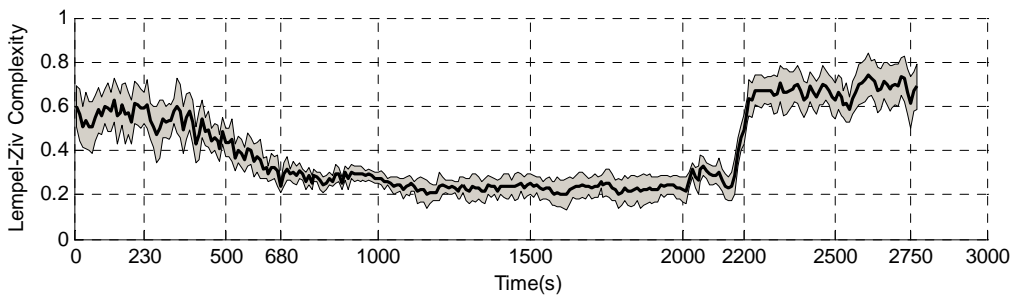

Fig. (2). The values of complexity measure $L Z C$ change against time during the whole anesthesia process of $16 \mathrm{channels.} \mathrm{The} \mathrm{black} \mathrm{thick}$ solid line in the middle represents the mean LZC extracted from LFP signals of 16channels. Gray area describes the variation range of $L Z C$ for all 16channels. In the whole experiment process, the 'light' anesthesia phase was the period of about 0s-600s after supplement anesthesic and the 'deep' anesthesia phase was the period of about $600 \mathrm{~s}-2100 \mathrm{~s}$, then recovery phase $(2100 \mathrm{~s}-)$ is also consider as 'light' anesthesia state.

where $P(f)$ is the estimate of power spectral density, $f$ is the corresponding frequency value, $f_{\min }$ and $f_{\max }$ are the minimum frequency and the maximum frequency value of LFP signals respectively. $f_{S E F}$ is the spectral edge frequency and $f_{G F}$ is the center frequency values, for the gravity frequency.

Approximate entropy(ApEn), a measure of signal complexity and regularity, quantifies LFP signals changes during anesthesia. A more detailed description of the PE calculation can be found in the published works [34].

The results of three indexes of anesthesia depth(SEF, GF and ApEn) derived from the V1 recordings were presented in Fig. (3). The trends of three time-varying indexes are coinci- dent with each other at each study period. In the start period after supplement anesthesic, three indexes tended to decrease similarly, and then remained steady-state. At last, the indexes increased rapily at the recovery phase. However, higher coherence existed between ApEn and LZC with the increasing deep anesthesia. The SEF and GF values had not the best correlation with anesthetic degree. The greatest disadvantage of ApEn is its slow computational speed which can't be satisfied with the requirement of real-time monitoring. The average computational time of ApEn value derived from 10 s recordings is about 7.29 second and the calculated time of LZC value at the same condition is about $0.12 \mathrm{~s}$. Therefore, we adopted complexity measure LZC Value as characteristic extracted from LFP to represent anesthesic degree in this study. 


\subsection{Predictive Effect of Anesthesia Depth with the Pre- dicting Model Based on SVM}

In order to assess the predictive performances for anesthesia depth, results from three different detection schemes are listed in Table 1. The accuracy is the lowest (92.08\%) when only using the optimal complexity threshold of raw LFPs. If we use an input vector consisting of an $L Z C$ of raw LFP and six LZC values of different frequency components for the SVM to predict anesthesia depth, the accuracy is
$95.68 \%$. On the basis of the seven characteristics discussed above, two frequency domain parameters ( $\delta$ ratio and $\beta$ ratio values) were introduced into the input vector of the SVM model; the highest accuracy achieved was $97.84 \%$ with the proper selection of kernel function and optimal parameters ( $C$ and $g$ ).

The SVM model worked well in tracking the same rat's anesthesia depth and could also provide the replenishment time of the anesthetic and proper period of data analysis in

Table 1. Comparison of Performances of Different Detection Schemes for Six LE Rats

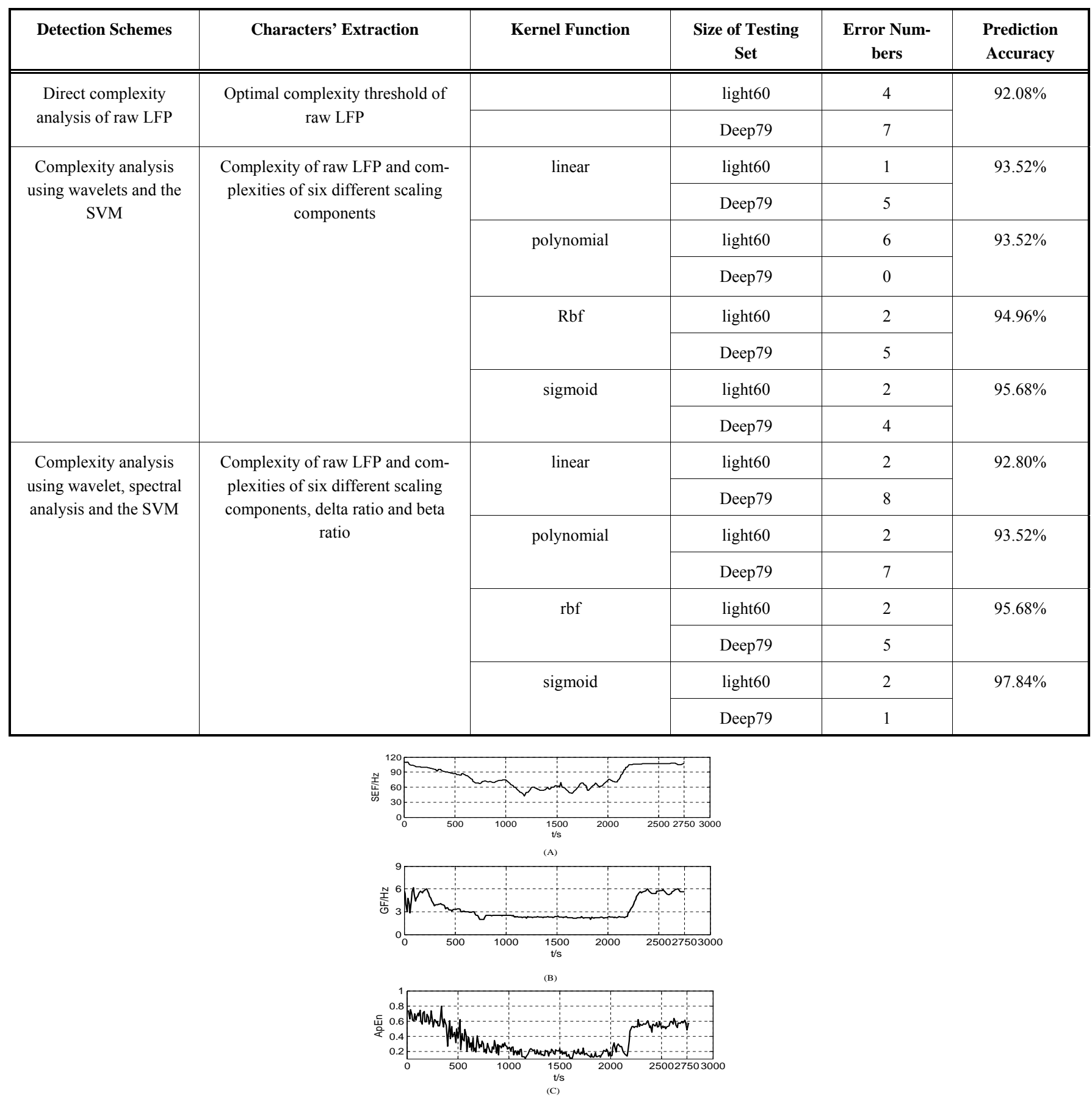

Fig. (3).Three indexes of anesthetic depth derived from the V1 recordings during the whole anesthesia process, (A) Time-varying SEF (B) Time-varying GF and (C) Time-varying ApEn 
Table 2. Comparison of Orientation Selectivity of the Neurons under Different Anesthesia Depth

\begin{tabular}{|c|c|c|c|c|c|c|c|c|c|c|c|c|c|}
\hline $\begin{array}{l}\text { Channel } \\
\text { Number }\end{array}$ & \multicolumn{3}{|c|}{ Slight (Label 0) } & \multicolumn{3}{|c|}{ Deep (Label 1) } & $\begin{array}{l}\text { Channel } \\
\text { Number }\end{array}$ & \multicolumn{3}{|c|}{ Slight (Label 0) } & \multicolumn{3}{|c|}{ Deep (Label 1) } \\
\hline 1 & Non & 2 & 0.50 & $270^{\circ}$ & 4.5 & 0.87 & 9 & $210^{\circ}$ & 17 & 0.89 & Non & 1 & 0.33 \\
\hline 2 & Non & 6 & 0.48 & $90^{\circ}$ & 6 & 0.71 & 10 & Non & 8 & 0.23 & Non & 1 & 0 \\
\hline 3 & $120^{\circ}$ & 4.5 & 1.0 & Non & 1 & 0.06 & 11 & $90^{\circ}$ & 15 & 0.82 & Non & 0.5 & 0 \\
\hline 5 & Non & 2 & 0.23 & Non & 1 & 0 & 13 & $120^{\circ}$ & 21.5 & 0.81 & Non & 0.5 & 0 \\
\hline 6 & $120^{\circ}$ & 22 & 1.0 & Non & 1.5 & 0 & 14 & $120^{\circ}$ & 26 & 1.0 & Non & 2 & 0 \\
\hline 7 & $210^{\circ}$ & 8 & 0.88 & Non & 0.5 & 0 & 15 & $240^{\circ}$ & 26 & 0.75 & $270^{\circ}$ & 7.5 & 0.87 \\
\hline 8 & $210^{\circ}$ & 9 & 0.71 & Non & 1 & 0 & 16 & Non & 4 & 0.48 & Non & 2 & 0 \\
\hline
\end{tabular}

Note: Ori represents optimal orientation; Fre is maximum firing rate in the optimal orientation; OSI means orientation selectivity index.

\section{Predicting Depth of Anesthesia}

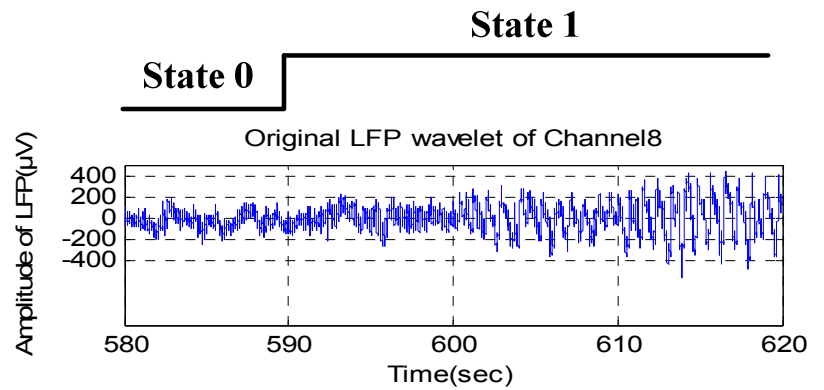

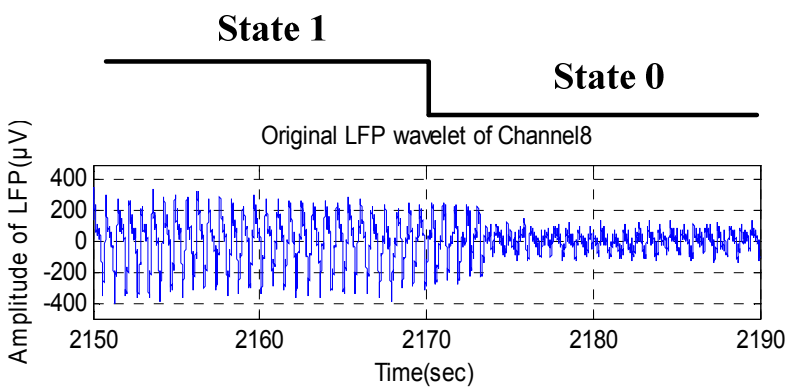

Fig. (4). State-turning between two kinds of anesthesia states, (A) Change from light anesthesia state to deep state. (B) Change from deep anesthesia state to light state.

visual stimulus experiments. Fig. (4) shows the conversion between two kinds of anesthesia states. To balance interference-resistance and sensitivity, switching of states requires that the same state is predicted 2-3 times. It can be seen that the anesthesia state-turning points will be predicted 10-20s in advance, which is largely predicted by the SVM model.

\subsection{The Influence of Anesthesia Depth on Visual Stimu- lation Experiments}

The visual stimulation experiments were carried out in order to analyze the influence of visual perception information transfer and processing. The sinusoidal drifting grating with 12 different orientations (in step of $30^{\circ}$, spatial frequency of 0.1 cycles $^{\circ}$, random play) was adopted as visual stimulus. A grating stimulus and gray screen each lasted 1s. Each test sequence was repeated 30 times in the same condition. Offline analysis was performed using Matlab. The single unit activity (spike) was obtained using band-pass filtering between $0.25-7.5 \mathrm{kHz}$, digitized at $30 \mathrm{kHz}$, threshold detecting and spike sorting in the pretreatment.

Firstly, 240 segments of LFP were collected in four different conditions, including spontaneous LFP signals in the light anesthesia state, spontaneous LFP signals in the deep anesthesia state, and stimulus evoked LFP signals in two different anesthesia states, respectively. There was a statistically significant difference $(\mathrm{P}<0.05)$ in complexity measured LZC between the light anesthesia and deep anesthesia state. However, there was no significant difference $(\mathrm{P}>0.05)$ in LZC computed from spontaneous LFP signals and stimulus evoked LFP signals in the same anesthesia state. Mean group difference was very small (about 0.06) in the deep anesthesia state, while the mean group difference was larger (about 0.25 ) in the light anesthesia state.

On the other hand, the orientation selectivity of each unit was analyzed statistically under two kinds of the anesthesia depth. The results are presented in Table 2. Most neurons represented non-orientation selectivity under the deep anesthesia but had optimal orientation obviously in the light anesthesia state due to their enhancing firing rate. For example, the average firing rate of neuron 6 was very low and had non-orientation selectivity under the deep anesthesia state, but its firing rate significantly increased under the slight state. Its optimal orientation was $120^{\circ}$ and its orientation tuning curve is shown in Fig. (5). Further, the optimal orientation could be in error to a certain extent because the firing rate can be changed due to many causes, for example, whether or not the attention mechanism was taking place in the visual perception, the rat's physical condition, or other unknown factors. 


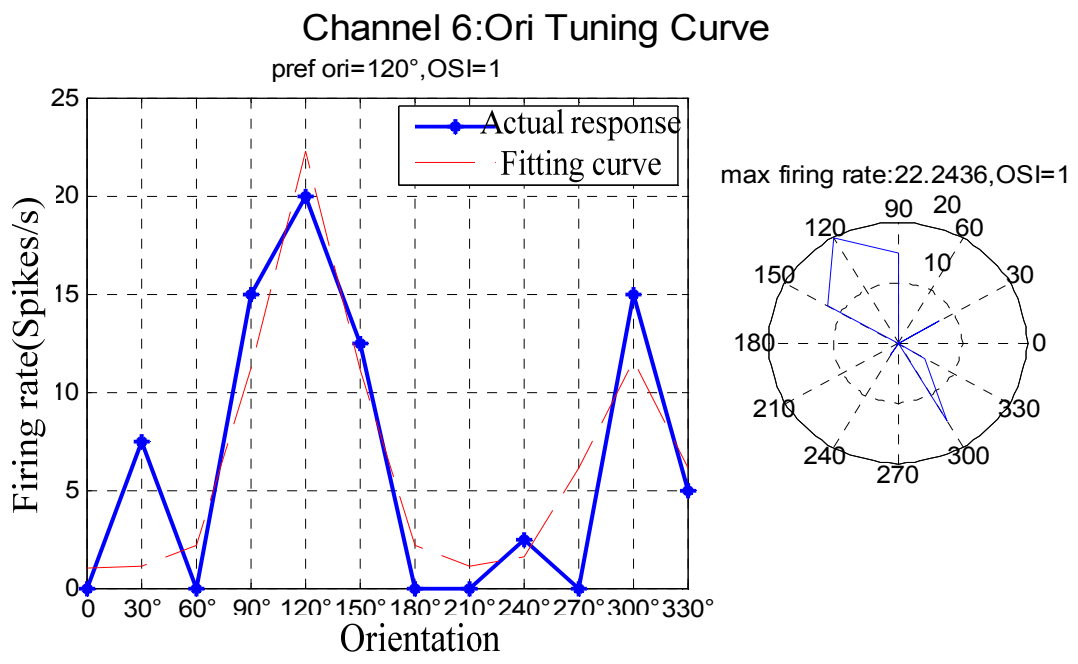

Fig. (5). Orientation Tuning Curve of channel6 under light anesthesia state.

The blue solid curve represents orientation tuning computed as the average firing rate evoked by sinusoidal drifting grating with 12 different orientations respectively from actual response of neuron by using the reverse correlation method. The detailed approaches are delineated in reference [33]. Individual orientation-tuning curve was fit to a two-peak Gaussian distribution (red dashed line), described in the following equation

$R(\theta)=K_{1} \times R_{\max } \times \exp \left[\frac{-\left(X-\theta_{\text {pref }}\right)^{2}}{2 \delta^{2}}\right]+K_{2} \times R_{\max } \times \exp \left[\frac{-\left(X-\theta_{\text {pref }}-180\right)^{2}}{2 \delta^{2}}\right]$

where $R_{\max }$ represents the maximum response rate, $K_{1}$ and $K_{2}$ are coefficients of responses, $X$ represents the orientations used, $\theta_{\text {pref }}$ represents the preferred orientation, $\delta$ represents the $\mathrm{SD}$, and baseline is the DC-offset of the Gaussian distribution.

To make quantitative comparisons of the degree of orientation selectivity for a neuron, we used the orientation selectivity index (OSI) defined as equation below

$O S I=\frac{\sqrt{\left[\sum R(\theta) \sin \theta\right]^{2}+\left[\sum R(\theta) \cos \theta\right]^{2}}}{\sum R(\theta)}$

where $\theta$ is stimulus orientation and $R(\theta)$ indicates the evoked peak response to each grating. A neuron that responds exclusively to a single orientation will have an OSI $=1$, whereas a neuron that responds equally to all orientations will have an OSI $=0$.

\section{DISCUSSION}

In this paper, a model based on the SVM was proposed to predict the anesthesia depth. First, the LFPs were decomposed into sub-waves in several frequency bands by wavelet transform. Then, some features representing the anesthesia states were extracted by nonlinear dynamic complexity analysis. The input vector of the model was composed by these features and two additional feature parameters in the frequency field. The experimental results of Long Evans rats demonstrated that the model could predict the anesthesia depth correctly through training samples and logical classification design in order to improve the adaptive ability of the model.

We have found several characteristics through analyzing the experimental data. With the deepening of the anesthesia, the LFPs recorded simultaneously in the same experimental animal tended to be more consistent with each other, and the low frequency components of the PSD were increased. While the correlation of complexity measures calculated from LFP of closely spaced intracortical sites (16 channels) was higher than the correlation of the raw LFPs of 16 channels, the threshold of $L Z C$ representing different anesthesia states would change in a certain range. This finding demonstrates that anesthetics coerce small cortical sub-networks, here represented by rats' primary visual cortex, into uniform, synchronized activity patterns. The complexity analysis of those sub-waves in each frequency bands showed that the complexity measure $L Z C$ in $\gamma$-band was more sensitive than other bands. As a result, the high frequency component $(\gamma-$ band) comprises important information of anesthesia states and is suitable for more accurate prediction. The predictive model has acquired more accuracy by taking into account the individuals' diversities. It is able to satisfy the monitory demand of the animals' anesthesia state during surgery and visual recording in vivo. This result is consistent with neurobiological research, in which the $\gamma$-band component of LFP plays an important role in the higher cognitive process of the brain, such as the receiving, encoding and processing of visual information in the brain's cortex [30]. The phenomenon that the anesthesia state has a predominant influence on the responses of visual stimulus experiments was found. The average firing rate of most neurons in the V1 area increased significantly in the light anesthesia state. These results imply that the anesthetic is likely to impair the visual information response and impede proper information exchange between cortical areas. Therefore, this model may be of great help in the selection of recorded data in visual experiments.

The predictive model proposed in this paper can be implemented more effectively due to the characteristics of LFP signals in time-frequency domain and special properties of 
neurons in the primary visual cortex. BIS is a monitoring anesthesia depth technology based on EEG, and its frequency band is narrow and not sensitive to noxious stimuli. However, the high frequency ( $>30 \mathrm{hz})$ components of LFP signals carry the finer information generally used to analyze anesthesia state. Two frequency domain parameters ( $\delta$ ratio and $\beta$ ratio values) used in BIS technology are retained in our model, and the LZC calculated from different frequency components of LFP can represent trends of anesthesia depth over time. Especially considering the properties of neurons in the primary visual cortex, the LZC of gamma frequency component should be significantly different in visual stimuli under the two different anesthesia states (light and deep). Therefore, we believe this model can improve the accuracy of prediction.

In this study, we only demonstrated the model's validity for ketamine hydrochloride administered intravenously by implanted MEA,but have not analyzed and compared the predictive effects for various anesthetics. In the future, we will conduct research on the anesthesia predictive model for different anesthetics' reaction, and then demonstrate the validity of the model for actual clinical impl

\section{CONFLICT OF INTEREST}

The authors confirm that this article content has no conflicts of interest.

\section{ACKNOWLEDGEMENT}

This study was supported by the National Natural Science Foundation of China (60971110, 6117215).

\section{REFERENCES}

[1] D. K. Christopher, and B. D. Karen, "Depth of anesthesia," Anesthesiology, vol. 22, pp.782-787, December 2009.

[2] D.R. Stanski, "Monitoring depth of anesthesia”. In: Anesthesia, R. D. Miller, Ed. Churchill Livingstone, New York, pp.1127-1159, 1994.

[3] R.E. Anderson, and J.G. Jakobsson, "Cerebral state monitor, a new small hand held EEG monitor for determining depth of anaesthesia: a clinical comparison with the bispectral index during daysurgery," Eur. J. Anaesthesiol., vol.23, pp. 208-212, 2006.

[4] V. Grover, and N. Bharti, "Measuring depth of anaesthesia-an overview on the currently available monitoring systems," Indian Anaesth',vol.1, pp.1-33, 2008.

[5] M.A. Rubin, and H. Freeman, "Brain potential changes in man during cyclopropane anesthesia," J. Neurophysiol., vol.3, pp.33-42, 1940.

[6] F. Tian, and Z. Ye. "A Review of the Application of Electroencephalogram In Detecting Depth of Anesthesia," J. Biomed. Eng., vol.22, pp.645-648, 2005.

[7] A. Sharma, and R.J. Roy, "Design of a recognition system to predict movement during anesthesia," IEEE Trans. Biomed. Eng., vol.44, pp.505-511, June 1997.

[8] J.C. Drummond, C.A. Brann, and D.E. Perkins, "A comparison of median frequency, spectral edge frequency, a frequency band power ratio, total power and dominance shift in the determination of depth of anesthesia," Acta Anaesthesiol. Scand., vol.35, pp. 693699,1991.

[9] Ben J. Palanca, George A. Mashour, Michael S. Avidan, "Processed electroencephalogram in depth of anesthesia monitoring,"Anesthesiology, vol.22, pp.553-559, October 2009.

[10] M.S. Avidan, L. Zhang, B.A. Burnside, K.J. Finkel, A.C. Searleman, J.A. Selvidge, L. Saager, M.S. Turner, S. Rao, M. Bottros, C.
Hantler, E. Jacobsohn, and A.S. Evers, "Anesthesia awareness and bispectral index," N. Engl. J. Med, vol. 358, pp.1097-1108, 2008.

[11] M.D. Kertai, B.J. Palanca, N. Pal, B.A. Burnside, L. Zhang, F. Sadiq, K.J. Finkel, and M.S. Avidan, B-Unaware Study Group. "Bispectral Index Monitoring, Duration of Bispectral Index Below 45, Patient Risk Factors, and Intermediate-term Mortality after Noncardiac Surgery in the B-Unaware Trial, ”Anesthesiology, vol.114, pp.545-556, 2011.

[12] S. Martin, K. Richard, and G. Matthias, "Comparison between bispectral index and patient state index as measures of the electroencephalographic effects of sevoflurane,"Anesthesiology., vol.109, pp. 799-805, 2008.

[13] X.S. Zhang, and R.J. Roy, "Predicting movement during anesthesia by complexity analysis of the EEG," Med. Biol. Eng. Comput., vol.37, pp.327-334, 1999.

[14] X.S. Zhang, and R.J. Roy, "Derived fuzzy knowledge model for estimating the depth of anesthesia," IEEE Trans. Biomed. Eng. vol.48, pp. 312-323, 2001

[15] C. Robert, J.F. and Gaudy, A. Limoge, "Electroencephalogram processing using neural networks," Clin. Neurophysiol., vol. 113, pp. 694-701, 2002.

[16] T. Gautama, D.P. Mandic, Van Hulle M.M. Van Hulle, "Indications of nonlinear structures in brain electrical activity," Phys. Rev., vol. 67. 046204, pp. 1-5, 2003.

[17] J. X. C. Zheng, and W. He, "Research on Approximate Entropy of Electroencephalogram for Monitoring Depth of Anesthesia," Space Med. Med. Eng., vol.17, pp. 205-209, 2004.

[18] L. Zhang, J. X. C. Zheng, G. Jing, and D. Lu, "Study on Kolmogorov Entropy of Electroencephalogram for Monitoring Depth of Anesthesia of Rat, "J. Xi'an Jiaotong Univ., vol.39,pp. 218-220, 2005.

[19] E. Olofsen, J.W. Sleigh, and A. Dahan, "Permutation entropy of the electroencephalogram: A measure of anaesthetic drug effect," $\mathrm{Br}$. J. Anaesth., vol.101, pp.810-821, 2008.

[20] A. Silva, A, H. Cardoso-Cruz, F. Silva, V. Galhardo, and L. Antunes, "Comparison of Anesthetic Depth Indexes Based on Thalamocortical Local Field Potentials in Rats, "Anesthesiology., vol. 112, pp.355-363, 2010.

[21] M. Kreuzer, H. Hentschke, B. Antkowiak, C. Schwarz, E.F. Kochs. and G. Schneider, "Cross-approximate entropy of cortical local field potentials quantifies effects of anesthesia - a pilot study in rats," BMC Neurosci., vol.11, pp. 122-133, 2010.

[22] A.G. Hudetz, "Effect of volatile anesthetics on interhemispheric EEG crossapproximate entropy," Brain Res.,vol.954, pp.123-131, 2002.

[23] A.G. Hudetz, J.D. Wood, and J.P. Kampine, "Cholinergic reversal of isoflurane anesthesia in rats as measured by cross-approximate entropy of the electroencephalogram," Anesthesiology, vol.99, pp.1125-1131, 2003.

[24] Xiaomei Du. "Application Progress of Electroencephalogram in Monitoring Depth of Anesthesia," Med. Recapitulate, vol.13, pp.1831-1833, December 2007.

[25] Z. Feng, and X. Zheng, "The Dynamic Change of Rat EEG's Complexity and Power Spectrum under Different Anesthetized Depth," Chin. J. Biomed. Eng., vol. 23, pp. 87-91,86, 2004.

[26] J. Xu, C. Zheng, X. Liu, and W. He, "Brain Activity Complexity Analysis to Rat Under Different Depth of Anesthesia," J. Xi'an Jitao Tong Univ., vol. 40, pp.739-744, 2006.

[27] A. Lempel , and J. Ziv, "On the complexity of finite sequences," IEEE Trans. Inform. Theory., vol.22, pp.75-81, 1976.

[28] G.Buzsáki, "The structure of consciousness," Nature, vol. 446, pp. 267, 2007.

[29] A. MT, H. AG, and T. G, "Consciousness and anesthesia," Science, vol. 322, pp.876-880, 2008

[30] P. Berens, G.A. Keliris, A.S. Ecker, N.K. Logothetis and A.S Tolias, "Feature selectivity of the gamma-band of the local field potential in primate primary visual cortex," Front in Neurosci., vol. 2, pp.199-207, 2008.

[31] B Yu,"Ideal anesthesia state and the depth of anesthesia monitoring,"Mod. Clin. Med. Biol. Eng. Mag., vol.12, no. 4, pp.305-307, 2006. 
[32] W.L. Mack, and J.W. Nelson, "A method of evaluating local anesthetics," Pharma Associ, vol.42 no, 2. pp.101-103, 1953.

[33] G. Felsen, Y. Shen, H. Yao, G. Spor, C. Li, and Y. Dan, "Dynamic Modification of Cortical Orientation Tuning Mediated by Recurrent Connections," Neuron, vol.36, pp.945-954, 2002
[34] J. Bruhn, H. Röpcke, B. Rehberg, T. Bouillon, and A. Hoeft, "Electroencephalogram approximate entropy correctly classifies the occurrence of burst suppression pattern as increasing anesthetic drug effect", Anesthesiology, vol.93, no. 4, pp.981-985, October 2000.

Received: March 05, 2013

Revised: April 26, 2013

Accepted: May 23, 2013

(C) Shi et al.; Licensee Bentham Open.

This is an open access article licensed under the terms of the Creative Commons Attribution Non-Commercial License (http://creativecommons.org/licenses/by-nc/3.0/) which permits unrestricted, non-commercial use, distribution and reproduction in any medium, provided the work is properly cited. 